\title{
Sistemas de transmisión del conocimiento etnobotánico de plantas silvestres comestibles en Turbo, Antioquia, Colombia.
}

\section{Transmission systems of Ethnobotanical knowledge of edible wild plants in Turbo, Antioquia, Colombia.}

\author{
Sistemas de transmissão de conhecimento etnobotânico de \\ plantas silvestres comestíveis em Turbo, Antioquia, Colômbia.
}

\author{
Ramón Antonio Mosquera Mena', Tomas Santamaría Polo² \& Juan Carlos López Almansa³ \\ ${ }^{1}$ Ingeniero Agroforestal, Especialista el Pedagogía para el Desarrollo del Aprendizaje Autónomo, \\ Magister en educación con énfasis en Educación Virtual, Doctor en Desarrollo Sostenible. ${ }^{2}$ Técnico \\ en Medio Ambiente, Magister en Gestión Ambiental, Doctor en Ciencias Biológicas. \\ ${ }^{3}$ Ingeniero de Montes, Doctor en Ciencias Biológicas. \\ ${ }^{1,3}$ Facultad de Desarrollo Sostenible. ${ }^{2}$ Vicerrectoría de doctorado y posgrado. \\ 1,2,3 Universidad Católica de Ávila. España. \\ 1'ramosquera01@gmail.com, ${ }^{2 j}$ carlos.lopez@ucavila.es, tomas.santamaria@ucavila.es
}

\begin{abstract}
Resumen
Los diferentes estudios etnobotánicos y etnoecológicos, muestran no solo la gran riqueza de recursos naturales que muchas comunidades han aprovechado de manera sostenible en el tiempo, si no también, la preocupación por la falta de sistematización del conocimiento debido a diferentes situaciones que lo dificultan. Esta investigación tiene como propósito mostrar los diferentes sistemas de transmisión del conocimiento que los habitantes del municipio de Turbo, Antioquia, Colombia, han utilizado para que las generaciones presentes y futuras reconozcan las plantas silvestres comestibles, lo cual es útil para tomar decisiones frente a la necesidad de sistematización del conocimiento de la biodiversidad local. Se utiliza una metodología que comprende las etapas de diseño de la encuesta etnobotánica, la aplicación de la misma teniendo en cuenta el tamaño de la población lo que arroja un total de 360 encuestas aplicadas en
\end{abstract}

los 18 corregimientos del municipio, agrupados en cuatro sectores fisiográficos: Serranía, Litoral, Abanico Aluvial y Planicie Inundable. Los resultados muestran las formas de transmisión de conocimiento en cada uno de los corregimientos que componen la geografía del territorio, evidenciando que continúan primando sistemas de transmisión oral con muy baja sistematización y con un desinterés de la población joven por reconocer dichos recursos orientados a la preservación como fuente de alimento.

Palabras clave: Etnobotánica, plantas silvestres, plantas comestibles, biodiversidad.

\section{Abstract \\ Diverse ethno-botanical and ethno-ecological stu- dies, have showed not only the wealth of natu- ral resources that many communities have taken}


advantage of them sustainably over time, but also, the concern for the lack of systematization of the knowledge due to different situations that make difficult it. This research aims to show the different systems of transmission of knowledge which have been used by the inhabitants of the municipality of Turbo, Antioquia, Colombia, so that present and future generations can recognize edible wild plants, useful to make decisions demonstrating the necessity of systematization of the knowledge of the local biodiversity. It was used a methodology that includes the stages of design of the ethno-botany survey, their application taking into account the size of the population resulting in a total of 360 surveys implemented in the 18 districts of the municipality, grouped into four physiographic areas: mountain, coast, alluvial fan and flood plain. Results showed the forms of transmission of knowledge in each of the jurisdictions that make up the geography of the territory, demonstrating that they continue giving priority to systems of oral transmission with low systematization and also there is a disinterest of youth recognizing these resources as a source of food preservation-oriented.

Keywords: Ethno-botany, wild plants, edible plants, biodiversity.

\section{Resumo}

Diferentes estudos etnobotânicos e etnobiológicos mostram não apenas a grande riqueza dos recursos naturais que muitas comunidades têm aproveitado de forma sustentável ao longo do tempo, mas também a preocupação pela falta de sistematização do conhecimento em razão de diferentes situações que o tornam difícil. Esta pesquisa tem como objetivo mostrar os diferentes sistemas de transmissão do conhecimento que os moradores do município de Turbo, Antioquia, Colômbia, têm utilizado para que as gerações presentes e futuras reconheçam as plantas selvagens comestíveis, o qual é útil na tomada de decisões perante a necessidade de sistematizar o conhecimento sobre a biodiversidade local. É usada uma metodología que envolve as etapas de delineamento e execução de entrevistas etnobotânicas, as quais levaram em consideração o tamanho da população, tendo um total de 360 entrevistas realizadas, em 18 bairros do município, agrupados em quatro setores fisiográficos: Serrania, Litoral, Abanico aluvial y Planície inundable. O estudo mostra as formas de transmissão do conhecimento em cada um dos bairros que compõem a geografía do território, mostrando que continuam prevalecendo os sistemas de transmissão oral com pouca sistematização e falta de interesse da população jovem em reconhecer ditos recursos como fonte de alimento.

Palavras-chave: Etnobotânica, plantas selvagens, plantas comestíveis, biodiversidade.

\section{Introducción}

La Etnobotánica se considera como la ciencia que estudia las relaciones que existen entre las plantas y el ser humano, el objetivo de ésta, es indagar el conocimiento que poseen algunos grupos sobre las propiedades de las plantas que se encuentran cerca a sus comunidades y que son utilizadas en sus hogares para diferentes fines (Ansalani, 2001).

El término etnobotánica, ha pasado de conocerse como el estudio del conocimiento ecológico y la forma de adaptación y modificación del hábitat de las plantas, al estudio de conocimientos ecológicos locales; debido a que en las investigaciones actuales la etnobotánica se focaliza en temas como: sistemas locales de conocimiento ecológico, relaciones entre diversidad biológica y diversidad cultural, entre otros (Cerón, 1999), estos estudios incluyen información sobre el clima, la flora y la fauna, referenciando los cambios que estos sistemas han tenido con la evolución de la humanidad (Phillips \& Gentry, 1993) . 
Hoy en día son muchas las investigaciones etnobotánicas que se adelantan en diferentes partes del mundo, con el propósito de conocer cómo el hombre utiliza los recursos naturales para su supervivencia, identificando y usando especies vegetales para alimento, ornamentos, industria, medicina, entre otros, logrando con estas prácticas el desarrollo sociocultural de sus comunidades.

El conocimiento que poseían las civilizaciones primitivas sobre el uso de las especies vegetales ha pasado de generación en generación, a través de enseñanzas, generalmente de padres a hijos; la utilización de prácticas tradicionales y el uso y manejo de los recursos naturales es de gran importancia para realizar investigaciones más profundas y verificables a partir de las formas en que los pueblos tradicionales se han relacionado con la naturaleza mediante creencias, conocimientos y prácticas, sin ocasionar alteraciones representativas al medio ambiente (Trujillo, 2010).

Los aportes de la etnobotánica, también hacen énfasis en el rescate de los saberes tradicionales como base para nuevos elementos de estudios orientados a la botánica económica, fitoquímica, biotecnología, entre otras disciplinas (Ramos, Ávila \& Morales, 2007; Berlin, Breedlove \& Raven, 1966). Lo que esta interdisciplina propone, es recuperar la estabilidad ecológica a partir de las técnicas que utilizan los agricultores tradicionales para manejar, conservar el suelo y los recursos naturales, con fin de contrarrestar los efectos secundarios que está padeciendo la población actual a causa de las alteraciones ambientales que el hombre ha provocado por las malas prácticas en la utilización de los recursos naturales (Ramos, Ávila \& Morales 2007; Lee, 1979).

Lo más destacable de la etnobotánica, es su dedicación a la recuperación y estudio del conocimiento que las sociedades, etnias y culturas de todo el mundo, poseen sobre las propiedades de las plantas y su utilización en todos los ámbitos de la vida. La información que aporta esta ciencia, va más allá del estudio del entorno natural, ya que además procura comprender su objeto de estudio en tanto patrimonio etnográfico cultural, material e inmaterial, resultando de gran utilidad y aplicación para el cuidado y conservación de los recursos fitogenéticos ligados a la subsistencia de los pobladores, el fortalecimiento y desarrollo de comunidades locales $y$, en particular, del área de la salud, cuando involucra el estudio de las plantas medicinales (Usma, 2003).

En los países de América Latina, la etnobotánica ha sido útil para el aprovechamiento de especies cultivadas a nivel mundial, algunas de ellas son tomate (Solanum lycopersicum), calabaza (Cucúrbita pepo L), maíz (Zea mays), aguacate (Persea americana), guanábana (Annona muricata), que debido a las condiciones ambientales presentes en estas zonas del mundo, permitieron el desarroIlo de estas plantas silvestres, las cuales han sido aprovechadas como alimentos, preparadas y consumidas de diversas formas y también en usos medicinales de vital importancia para las poblaciones alejadas de las comunidades o en zonas donde no había asistencia médica.

Conforme a lo anterior, puede notarse cómo diferentes autores se han dedicado a la realización de estudios etnobotánicos tendientes a la sistematización y visualización del conocimiento tradicional de diferentes poblaciones, como el caso de Yaroslava, (2002) quien adelantó en la sierra norte de México un estudio en el cual por medio del uso de entrevistas abiertas a agricultores, encontró más de 20 especies que guardan reservas subterráneas utilizadas por los pobladores con regularidad en su alimentación, reseñando que esta reserva es usada prioritariamente en épocas de escasez de otros alimentos tradicionales; dichas especies se encuentran en diferentes ecosistemas y aportan especialmente carbohidratos .

Continuando en México, como uno de los países de América que publica mayor cantidad de estudios en esta materia, se encuentra que Díaz \& Gispert (2000), relacionan en una región de Veracruz (Balzapote), las especies que la comunidad reconoce 
y usa en la alimentación y analizan los cambios que se presentan en dicha comunidad debido a la transformación de la estructura ecosistémica y la introducción de otros productos en la dieta común; en tal sentido, su estudio identifica 106 especies de uso alimenticio, las cuales corresponden a 92 géneros y 47 familias botánicas, éstas se estudiaron en los siguientes biomas transformados: huertos: 105 especies, milpa: 8 y potreros: 11 especies.

De otro lado, un estudio de Correa (2004) realizado en la Amazonía ecuatoriana, muestra la identificación de 71 plantas de uso alimenticio en la comunidad Quichua del Río Yasuní, para lo cual entrevista a siete personas de la comunidad, consideradas como expertas y utiliza parcelas demostrativas para la identificación de las plantas encontradas.

Este conocimiento tradicional identificado en el área, es transmitido de generación en generación a través de sistemas poco desarrollados, con muy poca sistematización y con alto grado de posibilidad de pérdida ya que depende de la capacidad de la comunidad para llevar la información de voz a voz (Granzow de la Cerda, 1993).

\section{Metodología}

El municipio de Turbo se encuentra localizado al norte del departamento de Antioquia (Colombia), en la llamada zona del Urabá antioqueño. El municipio se encuentra bañado por el mar Caribe y el río Atrato. Tiene una extensión de 3.055 km². Limita al norte con el mar Caribe y el municipio de Necoclí; al este con los municipios de San Pedro de Urabá y Apartadó, y con el departamento de Córdoba; al sur con los municipios de Carepa, Chigorodó y Mutatá, y al oeste con el departamento del Chocó como se aprecia en la Figura.1.

Su población total es de 122.780 habitantes (47.747 en la zona urbana) (DANE 2005) Se encuentra a una altitud promedio de $2 \mathrm{msnm}$ con una temperatura media de $28^{\circ} \mathrm{C}$ y una precipitación media anual de $1.925 \mathrm{~mm}$.

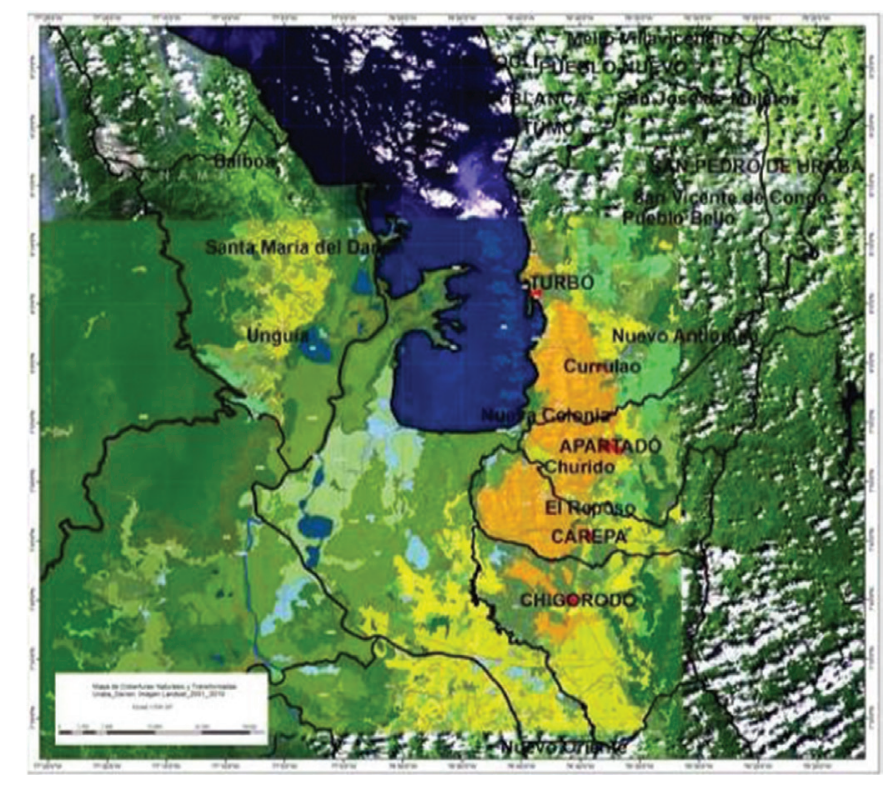

Figura 1. Avances del cambio de Uso del Suelo municipio de Turbo. Fuente, CORPOURABA 2009

La investigación se desarrolló en varias etapas, la primera de ellas consistió en el diseño de una encuesta aplicada a los recolectores de plantas silvestres comestibles de los 18 corregimientos que componen el municipio de Turbo, Antioquia (El tres, Rio Grande, Nueva Colonia, Punta de piedra, El dos, Bocas de Atrato, Turbo cabecera, Currulao, Alto de Mulatos, Nuevo Antioquia, Pueblo 
bello, San José, San Vicente, Blanquicet, Lomas aisladas, Macondo, Nuevo oriente y Puerto rico) con la cual se obtuvo tanto información para la clasificación de los informantes, con base a criterios de etnia, sexo, edad y oficio; como información relacionada con las plantas, partes comestibles, técnicas, sistemas de transmisión del conocimiento, también información sobre la persona que enseña sobre la planta y modos de recolección y manejo así como los modos de preparación y conservación de alimentos. Como complemento al trabajo de encuestas se realizaron visitas de campo para la verificación de especies y recolección de muestras para una identificación fiable de las mismas así como la confección de un herbario etnobotánico.

\section{a. Diseño del cuestionario.}

El cuestionario diseñado para la encuesta de esta investigación, además de identificar la planta y conocer su uso, permite obtener información etnocultural y relacionar y clasificar la información botánica bajo parámetros sociológicos y culturales. El modelo de encuesta se basa en trabajos similares tanto latinoamericanos (Cerón, 1999; Usma, 2003; González Insuasti, 2006; Trujillo, 2010), como españoles (Granzow de la Cerda, 1993; Tardío et al., 2006; Díaz Fernández et al., 2009).

\section{b. Aplicación de la encuesta.}

Se entrevistaron personas adultas, mayores de 18 años hombres y mujeres, por ser éstos los responsables de la alimentación de la familia. Teniendo en cuenta que según la base de datos del SISBEN (2011), en el municipio de Turbo, la población total es de 144.281 habitantes, de los cuales 79.452 habitantes corresponden a población mayor de 18 años, esto es un $55 \%$ de la población total y que de esta población adulta se encuentran entre los 18 y 30 años 39.555 habitantes; entre 31 y 45 años 22.132 y mayores de 45 años 17.765 habitantes, a partir de la población total de 79.452 se aplicó la fórmula de cálculo de muestra para poblaciones finitas propuesta por González \& Caballero (2006), obteniendo una muestra de 320 encuestas con un error de 5.5\%.
Una vez seleccionada la muestra, se procedió a la aplicación de la encuesta en los 18 corregimientos que componen el municipio de Turbo. Se aplicaron 20 encuestas por corregimiento, se dividió la muestra en personas entre los rangos de edad 18 a 40 años, 41 a 60 años y más de 61 años. La proporción de aplicación fue de $25 \%$ para el primer grupo de edad, $50 \%$ para el segundo y $25 \%$ para el tercer grupo.

\section{c. Procesamiento de los datos.}

Los datos obtenidos fueron procesados con el fin de obtener valores medios y datos porcentuales así como contrastar la información relacionada con la persona que enseña el conocimiento de la planta de acuerdo al sector fisiográfico de los corregimientos del municipio de Turbo que les corresponda: Serranía (San José, San Vicente del Congo, Pueblo Bello, Alto de Mulatos, Nuevo Antioquia y Currulao); Litoral (Tie, El Dos, Bocas de Atrato, Turbo cabecera); Abanico Aluvial (El tres, Río Grande, Nueva Colonia y Planicie Inundable (Lomas Aisladas, Blanquicet, Macondo, Puerto rico, Nuevo oriente).

Para el tratamiento estadístico se asumió cada espacio fisiográfico como un bloque, que estuvo integrado por 20 encuestas por corregimiento, tomando cada corregimiento como una repetición, se procesaron los resultados y se compararon entre los espacios fisiográficos para establecer diferencias significativas entre los sectores y al interior de cada uno de ellos aplicando un análisis de varianza de una vía.

\section{Resultados}

Se encontraron 1.280 datos válidos para plantas silvestres comestibles en los 18 corregimientos que componen el municipio de Turbo, Antioquia, Colombia, los cuales en cuanto al sistema oral de transmisión del conocimiento etnobotánico, muestran que los encuestados han recibido el conocimiento con la siguiente descripción: 310 
datos el conocimiento fue transmitido por el padre, correspondiente al $24 \%$ de los datos suministrados; 494 datos son trasmitidos por la madre, correspondiente al 39\%; 340 datos muestran que la transmisión del conocimiento se produce por los abuelos, lo que corresponde al $26 \%$ y 136 datos, correspon- den a conocimiento suministrado por amigos, lo cual corresponde a un $11 \%$ del total de datos, como se muestra en la Tabla 1 la cual evidencia el inventario de plantas identificadas por los encuestados como silvestres comestibles, la cantidad de registros y la persona que transmitió el conocimiento sobre ella.

Tabla 1. Inventario de plantas silvestres comestibles y transmisión de conocimiento del municipio de Turbo, Antioquia, Colombia

\begin{tabular}{|c|c|c|c|c|c|c|c|c|c|}
\hline \multirow{2}{*}{ No. } & \multirow{2}{*}{$\begin{array}{l}\text { Nombre } \\
\text { común }\end{array}$} & \multirow{2}{*}{$\begin{array}{l}\text { Nombre } \\
\text { científico }\end{array}$} & \multirow{2}{*}{ Cantidad } & \multicolumn{6}{|c|}{ Quién le enseñó sobre la planta } \\
\hline & & & & Padre & madre & Abuelos & Tíos & Amigos & Otros \\
\hline 1 & Achiote & Bixa orellana & 23 & 4 & 6 & 2 & 4 & 3 & 4 \\
\hline 2 & Ají & $\begin{array}{l}\text { Capsicum } \\
\text { annuum }\end{array}$ & 8 & & 4 & 2 & 2 & & \\
\hline 3 & Aguacate & $\begin{array}{l}\text { Persea } \\
\text { americana }\end{array}$ & 28 & 3 & 6 & 4 & 3 & 10 & 2 \\
\hline 4 & Ahuyama & $\begin{array}{l}\text { Cucurbita } \\
\text { maxima }\end{array}$ & 34 & 6 & 12 & 6 & 4 & 6 & \\
\hline 5 & Algarrobo & $\begin{array}{l}\text { Ceratonia } \\
\text { siliqua }\end{array}$ & 8 & 2 & 5 & 1 & & & \\
\hline 6 & Almendra & $\begin{array}{l}\text { Terminalia } \\
\text { catappa }\end{array}$ & 18 & 3 & 8 & 2 & 4 & 1 & \\
\hline 7 & Anón & $\begin{array}{l}\text { Annona } \\
\text { squamosa }\end{array}$ & 30 & 5 & 13 & 4 & 7 & 1 & \\
\hline 8 & $\begin{array}{l}\text { Árbol del } \\
\text { pan }\end{array}$ & $\begin{array}{l}\text { Artocarpus } \\
\text { altilis }\end{array}$ & 24 & 8 & 12 & 2 & 2 & & \\
\hline 9 & Badea & $\begin{array}{l}\text { Passiflora } \\
\text { quadrangularis }\end{array}$ & 24 & 7 & 11 & 4 & 1 & 1 & \\
\hline 10 & Batata & $\begin{array}{l}\text { Ipomoea } \\
\text { batatas }\end{array}$ & 24 & 8 & 12 & & 1 & 3 & \\
\hline 11 & Berenjena & $\begin{array}{l}\text { Solanum } \\
\text { melongena }\end{array}$ & 22 & 5 & 10 & 3 & 1 & 3 & \\
\hline 12 & Bledo & $\begin{array}{l}\text { Amaranthus } \\
\text { tricolor }\end{array}$ & 17 & 8 & 9 & & & & \\
\hline 13 & Cacahuillo & $\begin{array}{l}\text { Theobroma } \\
\text { subincatum }\end{array}$ & 20 & 9 & 8 & & 1 & 2 & \\
\hline 14 & Caimitillo & $\begin{array}{l}\text { Chrysophyllum } \\
\text { cainito }\end{array}$ & 14 & 6 & 6 & & & 2 & \\
\hline 15 & Calabaza & $\begin{array}{l}\text { Cucurbita } \\
\text { moschata }\end{array}$ & 19 & 4 & 9 & 1 & 2 & 3 & \\
\hline 16 & Caña Agria & Costus spicatus & 32 & 5 & 12 & 6 & 2 & 5 & 2 \\
\hline 17 & Caña fistula & Cassia fistula $L$ & 30 & 7 & 13 & & 2 & 4 & 4 \\
\hline 18 & Carambolo & $\begin{array}{l}\text { Averrhoa } \\
\text { carambola }\end{array}$ & 32 & 8 & 14 & 4 & 2 & 4 & \\
\hline
\end{tabular}


...continuación Tabla 1

\begin{tabular}{|c|c|c|c|c|c|c|c|c|c|}
\hline \multirow{2}{*}{ No. } & \multirow{2}{*}{$\begin{array}{l}\text { Nombre } \\
\text { común }\end{array}$} & \multirow{2}{*}{$\begin{array}{l}\text { Nombre } \\
\text { científico }\end{array}$} & \multirow{2}{*}{ Cantidad } & \multicolumn{6}{|c|}{ Quién le enseñó sobre la planta } \\
\hline & & & & Padre & madre & Abuelos & Tíos & Amigos & Otros \\
\hline 19 & Caráño & $\begin{array}{l}\text { Protium } \\
\text { asperum }\end{array}$ & 13 & 4 & 8 & & & 1 & \\
\hline 20 & cepa & $\begin{array}{l}\text { Colocasia } \\
\text { esculenta }\end{array}$ & 36 & 6 & 17 & 4 & 5 & 2 & 2 \\
\hline 21 & Chascarrá & Bactris maraja & 15 & 4 & 9 & & 1 & 1 & \\
\hline 22 & Chirimoya & $\begin{array}{l}\text { Annona } \\
\text { cherimola }\end{array}$ & 28 & 8 & 14 & 3 & 1 & 2 & \\
\hline 23 & Churíma & Inga Fastuosa & 23 & 9 & 14 & & & & \\
\hline 24 & Cilantro & $\begin{array}{l}\text { Coriandrum } \\
\text { sativum }\end{array}$ & 24 & 7 & 12 & 2 & 1 & 1 & 1 \\
\hline 25 & Ciruela & $\begin{array}{l}\text { spondiam } \\
\text { purpurea }\end{array}$ & 27 & 8 & 13 & 2 & 3 & 1 & \\
\hline 26 & Col & $\begin{array}{l}\text { Brassica } \\
\text { oleracea }\end{array}$ & 18 & 6 & 9 & & 1 & 1 & 1 \\
\hline 27 & Corozo & $\begin{array}{l}\text { Bactris } \\
\text { guineensis }\end{array}$ & 31 & 9 & 14 & 4 & 3 & & 1 \\
\hline 28 & Espinaca & $\begin{array}{l}\text { Spinacia } \\
\text { oleracea }\end{array}$ & 22 & 5 & 11 & 3 & 1 & 2 & 1 \\
\hline 29 & Jengibre & $\begin{array}{l}\text { Zingiber } \\
\text { officinale }\end{array}$ & 26 & 7 & 8 & 6 & 1 & 2 & 2 \\
\hline 30 & Guama & $\begin{array}{l}\text { Inga Edulis } \\
\text { Mart }\end{array}$ & 23 & 8 & 6 & 5 & 3 & 1 & \\
\hline 31 & Guanábana & $\begin{array}{l}\text { Annona } \\
\text { muricata }\end{array}$ & 21 & 5 & 7 & 5 & 2 & 2 & \\
\hline 32 & Guayaba & $\begin{array}{l}\text { Psidium } \\
\text { guajava }\end{array}$ & 21 & 8 & 12 & 1 & & & \\
\hline 33 & Habichuela & $\begin{array}{l}\text { Phaseolus } \\
\text { vulgaris }\end{array}$ & 12 & 9 & 2 & 1 & & & \\
\hline 34 & Hiraca & $\begin{array}{l}\text { Carludovica } \\
\text { palmata }\end{array}$ & 27 & 6 & 3 & 6 & 8 & 4 & \\
\hline 35 & $\begin{array}{l}\text { Lechuga de } \\
\text { monte }\end{array}$ & Lactuca sativa & 15 & 4 & 5 & 4 & 2 & & \\
\hline 36 & $\begin{array}{l}\text { Limón } \\
\text { mandarina }\end{array}$ & Citrus $\times$ limonia & 13 & 5 & 4 & & 4 & & \\
\hline 37 & Limoncillo & $\begin{array}{l}\text { Cymbopogon } \\
\text { citratus }\end{array}$ & 36 & 7 & 7 & 5 & 9 & 6 & 2 \\
\hline 38 & Mafafa & $\begin{array}{l}\text { Xanthosoma } \\
\text { undipes Koch }\end{array}$ & 23 & 8 & 1 & 4 & 8 & & 2 \\
\hline 39 & Malanga & $\begin{array}{l}\text { Xanthosoma } \\
\text { sagittifolium }\end{array}$ & 22 & 4 & 7 & 2 & 6 & 1 & 2 \\
\hline 40 & Mamey & $\begin{array}{l}\text { Mammea } \\
\text { americana } L\end{array}$ & 29 & 6 & 9 & & 9 & & 5 \\
\hline 41 & Mamoncillo & $\begin{array}{l}\text { Melicoccus } \\
\text { bijugatus }\end{array}$ & 29 & 4 & 2 & 7 & 12 & & 4 \\
\hline
\end{tabular}


...continuación Tabla 1

\begin{tabular}{|c|c|c|c|c|c|c|c|c|c|}
\hline \multirow{2}{*}{ No. } & \multirow{2}{*}{$\begin{array}{l}\text { Nombre } \\
\text { común }\end{array}$} & \multirow{2}{*}{$\begin{array}{l}\text { Nombre } \\
\text { científico }\end{array}$} & \multirow{2}{*}{ Cantidad } & \multicolumn{6}{|c|}{ Quién le enseñó sobre la planta } \\
\hline & & & & Padre & madre & Abuelos & Tíos & Amigos & Otros \\
\hline 42 & Mango & $\begin{array}{l}\text { Manguifera } \\
\text { indica }\end{array}$ & 4 & 1 & 2 & & 1 & & \\
\hline 43 & Maracuyá & $\begin{array}{l}\text { Passiflora } \\
\text { edulis }\end{array}$ & 36 & 5 & 9 & 7 & 14 & & 1 \\
\hline 44 & Marañón & $\begin{array}{l}\text { Syzygium } \\
\text { malaccense }\end{array}$ & 24 & 4 & 12 & 8 & & & \\
\hline 45 & Naranjo & $\begin{array}{l}\text { Citrus } x \\
\text { sinensis }\end{array}$ & 21 & 8 & 7 & 5 & & & 1 \\
\hline 46 & Níspero & $\begin{array}{l}\text { Eryobotria } \\
\text { japonica }\end{array}$ & 18 & 3 & 9 & 4 & 2 & & \\
\hline 47 & Noni & $\begin{array}{l}\text { Morinda } \\
\text { citrifolia }\end{array}$ & 33 & 2 & 8 & 9 & 14 & & \\
\hline 48 & Ñame & Dioscorea spp & 28 & 7 & 6 & 6 & 2 & 6 & \\
\hline 49 & Orégano & $\begin{array}{l}\text { Origanum } \\
\text { vulgare }\end{array}$ & 21 & 3 & 8 & 4 & & 6 & \\
\hline 50 & Paico & $\begin{array}{l}\text { Chenopodium } \\
\text { ambrosioides }\end{array}$ & 8 & 4 & 4 & & & & \\
\hline 51 & $\begin{array}{l}\text { Palma de } \\
\text { vino }\end{array}$ & $\begin{array}{l}\text { Scheelea } \\
\text { butyracea }\end{array}$ & 25 & 4 & 9 & 7 & & 5 & \\
\hline 52 & Papaya & Carica papaya & 19 & 4 & 7 & 5 & 3 & & \\
\hline 53 & Papayuela & $\begin{array}{l}\text { Caica } \\
\text { pubescens }\end{array}$ & 7 & 1 & 3 & 3 & & & \\
\hline 54 & Tamarindo & $\begin{array}{l}\text { Tamarindus } \\
\text { indica }\end{array}$ & 12 & 2 & 8 & 1 & 1 & & \\
\hline 55 & Taparo & Attalea allenii & 28 & 5 & 9 & 4 & 5 & 5 & \\
\hline 56 & Tawa & $\begin{array}{l}\text { Beilschmiedia } \\
\text { tawa }\end{array}$ & 17 & 4 & 8 & 3 & 2 & & \\
\hline 57 & Toronjil & $\begin{array}{l}\text { Melissa } \\
\text { officinalis }\end{array}$ & 17 & 4 & 6 & 4 & 2 & 1 & \\
\hline 58 & uchuva & $\begin{array}{l}\text { Physalis } \\
\text { peruviana }\end{array}$ & 21 & 4 & 12 & 1 & & 4 & \\
\hline \multicolumn{3}{|c|}{$\begin{array}{l}\text { Totales Transmisión } \\
\text { del conocimiento }\end{array}$} & & 310 & 491 & 176 & 164 & 102 & 37 \\
\hline
\end{tabular}

Según los resultados mostrados en la Tabla 1 y al cruzarlos con cada sector fisiográfico del municipio se encuentra que el sector Serranía, aportó para el estudio el $33 \%$ de los datos, la Planicie Inundable el $28 \%$, el Litoral el $22 \%$ y el Abanico Aluvial el $17 \%$ de los datos, lo cual se puede apreciar en la Figura.2.

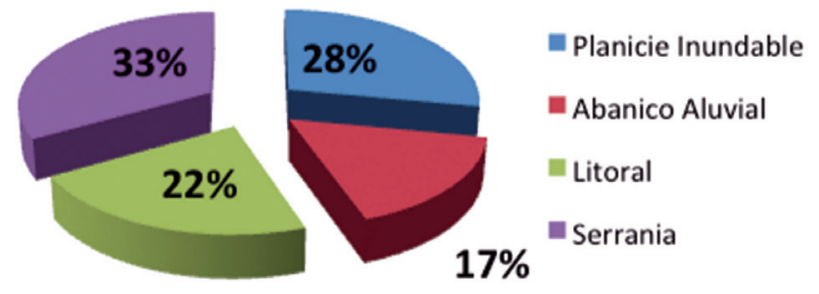

Figura 2. Transmisión del conocimiento Etnobotánico por sector fisiográfico en el municipio de Turbo, Antioquia 


\section{Análisis de transmisión del conocimiento por sector fisiográfico}

Al analizar el comportamiento de los datos del estudio y teniendo en cuenta la variabilidad por el número de corregimientos que componen cada espacio fisiográfico, se puede apreciar que en los espacios fisiográficos la transmisión del conocimiento se comporta de manera homogénea ya que la madre es la principal aportante, posteriormente el padre, seguidos por abuelos y amigos.

Al aplicar un análisis de varianza a la información se encuentra que hay diferencia significativa al comparar los espacios fisiográficos del municipio lo cual obedece a la cantidad de datos que se encuentran en cada uno, mas no a la distribución de los mismos entre el sector.

\section{Análisis sector Planicie Inundable}

El entorno fisiográfico correspondiente a la Planicie Inundable está constituido por los corregimientos de Blanquicet, Lomas aisladas, Macondo, Nuevo oriente, Puerto rico. En este espacio se encontró que la madre es transmisora del conocimiento en un $38 \%$ de los casos, el padre en un $24 \%$ de los casos, los abuelos en un $27 \%$ de los casos y los amigos en un $11 \%$ de los casos. Lo que se observa en la Figura 3

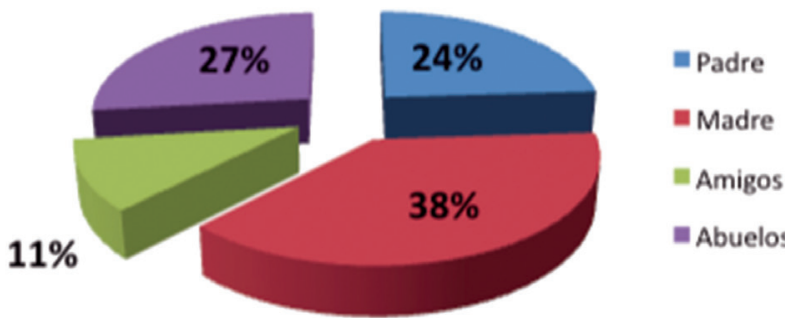

Figura 3. Transmisión del conocimiento Etnobotánico en el Sector Planicie Inundable del municipio de Turbo Antioquia - Colombia

El comportamiento de los datos en el sector, muestra irregularidad en la distribución de los mismos, especialmente entre los valores relacionados con abuelos y padres en la transmisión del conocimiento; y al aplicar un análisis de varianza para este sector, se encontró que hay diferencia significativa entre los corregimientos del sector.

Análisis sector Abanico Aluvial. Este sector está compuesto por los corregimientos de El tres, Rio Grande y Nueva Colonia, en ellos se encuentra que la madre es la mayor transmisora del conocimiento etnobotánico con un $39 \%$ de los datos suministrados, seguido por los abuelos con un $26 \%$, los padres con un $24 \%$ y amigos con un $11 \%$, como se aprecia en la Figura 4

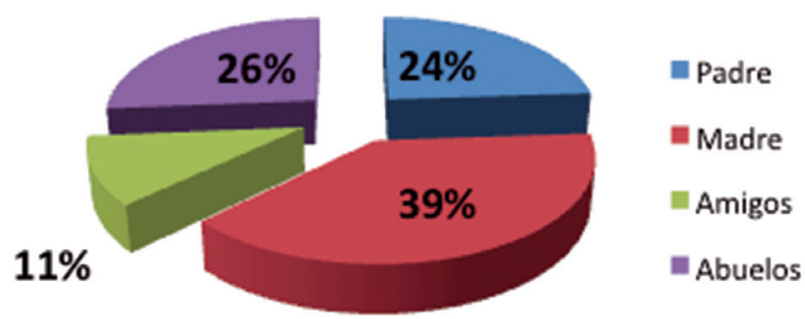

Figura 4. Transmisión del Conocimiento Etnobotánico Sector Abanico Aluvial del municipio de Turbo, Antioquia

Al analizar el comportamiento de los datos entre los corregimientos se encuentra que este sector muestra bastante homogeneidad en su distribución y al aplicarle el análisis de varianza a los datos se halla que no hay diferencias significativas entre los datos del sector.

Análisis sector Litoral. La Figura 5, muestra que en el sector Litoral, el conocimiento Etnobotánico es enseñado por la madre en un $39 \%$, los abuelos en un $26 \%$, los padres en un $24 \%$ y los amigos en un $11 \%$.

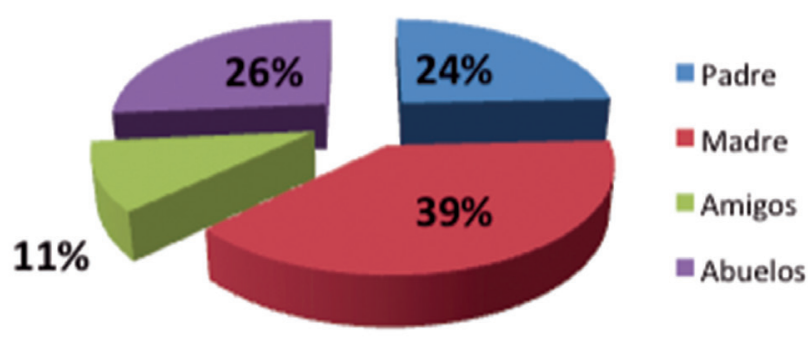

Figura 5. Transmisión del Conocimiento Etnobotánico Sector Litoral del municipio de Turbo, Antioquia 
Al analizar la dinámica de ocurrencia de los datos se encontró que el casco urbano de Turbo, muestra un comportamiento diferente a los demás corregimientos del sector Litoral, el cual está compuesto por los corregimientos de Punta de piedra, El dos, Bocas de Atrato y Turbo cabecera. Al aplicar el análisis de varianza de una vía, no se presentaron diferencias significativas en los datos suministrados.

Análisis sector Serranía. En este sector conformado por los corregimientos de Currulao, Alto de Mulatos, Nuevo Antioquia, Pueblo bello, San José y San Vicente, se encontró que la responsable mayoritaria de transmitir el conocimiento etnobotánico es la madre con un $38 \%$, seguido por los abuelos en un $27 \%$, los padres en un $24 \%$ y los amigos en un $11 \%$ como se aprecia en la Figura 6.
$11 \%$

Figura 6. Transmisión del Conocimiento etnobotánico Sector Serranía

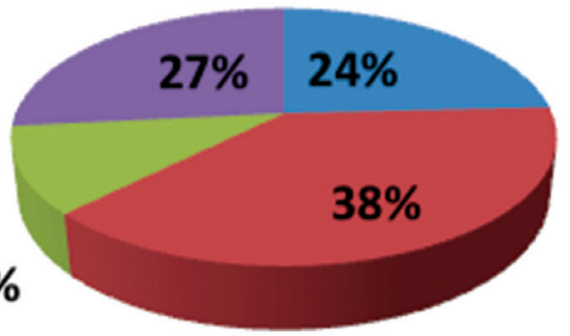

Padre

- Madre

Amigos

Abuelos conocimientos son transmitidos por la madre quien enseña no sólo las clases de plantas sino también las formas de preparación.

Al respecto se encuentra que Ramos et al. (2007), manifiestan que al realizar este tipo de estudios, se encuentra muy poca información, pero advierten la posibilidad de pérdida del conocimiento debido a la avanzada edad de los poseedores del mismo y al desinterés que presentan las nuevas generaciones por dicho conocimiento. Situación similar fue observada en el estudio de Turbo, en el cual la edad es directamente proporcional a la cantidad de información de plantas suministrada, haciendo necesaria la reflexión sobre el papel de las nuevas generaciones en la conservación de la información etnobotánica.

De igual manera las mujeres son poseedoras y transmisoras importantes del conocimiento etnobotánico, en plantas silvestres comestibles, lo cual se mantiene en este estudio donde las mujeres (madres) presentaron mayoría en la transmisión del conocimiento. Una probable razón puede ser la estructura matriarcal de los hogares en la zona de estudio, lo cual se manifiesta en la dependencia de la familia hacia la madre.

No se encuentran estudios que realicen comparaciones en diferentes sectores fisiográficos relacionados con procesos de transmisión del conocimiento, pero es importante mencionar que en esta investigación, la madre, en todos los sectores fisiográficos aparece como la transmisora principal del conocimiento. Una posible razón para este resultado puede deberse a que la mayoría de los corregimientos del municipio de Turbo, corresponde a áreas rurales, en las cuales la dinámica familiar implica que los niños se quedan en la casa con la madre mientras el padre está desempeñando labores en el campo y al estar ella encargada de la preparación de los alimentos en los cuales utiliza plantas silvestres, produce transmisión de conocimiento bien por curiosidad del aprendiente, bien por instrucción dada para enseñar diferentes procesos de preparación de alimentos. 
El riesgo de desaparición del conocimiento, es algo tangible y de consideración alta, debido a varios factores, entre los que se encuentran el cambio de vocación de los sitios de recolección y a que disminuyen las áreas de cosecha y las especies silvestres comestibles.

Lo anterior, demanda acciones preventivas y correctivas de carácter inter-institucional tendientes a la recuperación por un lado de las áreas degradadas, y por otro a la sistematización de la información relacionada con plantas silvestres comestibles, al mismo tiempo que se realiza el fomento de las características benéficas que tienen éstas en la alimentación como medida de preservación de los espacios de crecimiento para las mismas.

\section{Literatura citada}

1. Ansalani, R. (2001). Estudio de diversidad vegetal en el centro Shuar Yukutais, Morona Santiago, Ecuador. Revista de investigaciones de la Universidad del Azuay (Cuenca) 11: 30-52.

2. Berlin, B., Breedlove, D. E. \& Raven, P.H. (1966). Las taxonomías populares y clasificación biológica. Ciencia 154: 273-275.

3. Cerón, C.E. (1999). Etnobotánica Quichua del Río Yasuní, Amazonia Ecuatoriana.

4. CORPOURABA. (2009). Plan de Ordenamiento Territorial del Municipio de Turbo. Corporación Autónoma Regional para el Desarrollo Sostenible de Urabá.

5. Correa, M.E. (2004). Los recursos vegetales en el paisaje fragmentado generado por la agricultura itinerante: Un estudio de caso en el área Maya Yucateca de México. Tesis de pregrado: Licenciatura, Facultad de Ciencias, Universidad Nacional Autónoma de México, México, D.F., México.

6. DANE (2005). Censo poblacional de Colombia. Departamento Nacional de Estadísticas.

7. Díaz, A. \& Gispert, M. (2000). Cultura alimentaria de plantas cultivadas: un estudio de caso en Balzapote, Veracruz México. Etnobotánica y Botánica económica. 308

8. Díaz-Fernández, P.M.; Ramos Miras, J.J.; San José Wery, A.M.; López Almansa, J.C.; del Monte Maíz, M; \& Muñoz Gallego, C.L. (2009). Estudio etnoecológico de especies forestales comestibles en la Provincia de Ávila. 5 Congreso Forestal Español. Ávila.

9. Estupiñán-González, J.A (2010). Uso de las plantas por grupos campesinos en la franja tropical del parque nacional natural paramillo (Córdoba, Colombia). Recuperado de: www.unal.edu.co/icn/publicaciones/caldasia.htm
10. González Insuasti, M.S. (2006). "Etnobiología y sistemas biocognitivos tradicionales: paradigmas en la conservación biológica y el fortalecimiento cultural" En: Universidad Autónoma del Estado de Hidalgo, Asociación Etnobiológica Mexicana y Sociedad Latinoamericana de Etnobiología. México. (ISBN 978-607-482-095-9).

11. González-Insuasti, M. \& Caballero, J. (2006). Gestión de recursos de las plantas. Ecología Humana, 35: 303-314.

12. Granzow de la Cerda, I. (1993). Etnobotánica. El Mundo vegetal en la tradición. Salamanca. Centro de Cultura Tradicional, Diputación de Salamanca.

13. Lee, R.B. (1979). The IKung San: Hombres, mujeres y trabajo en un espacio de sociedad en New York: Cambridge University Press.

14. Pardo de Santayana, M. \& Gómez Pellón, E. (2003). Etnobotánica: Aprovechamiento tradicional de plantas y patrimonio cultural. Anales Jard ín Botánico de Madrid 60(1): 171-182.

15. Phillips, O. \& Gentry, A.H. (1993). Un estudio etnobotánico de la medicina tradicional de los pueblos mestizos de Suni Mirano, Loreto, Perú. Departamento de Botánica de la Universidad de British Columbia, 3515-6270 University Blvd., Vancouver, BC V6T 1Z4, Canadá.

16. Ramos Hernández, M., Ávila, C.H. \& Morales, J.E:(2007). Etnobotánica y ecología de plantas utilizadas por tres curanderos contra la mordedura de serpiente en la región de Acayucan, Veracruz, México. Boletín de la Sociedad Botánica de México, núm. 81, 89-100, Sociedad Botánica de México - México. Recuperado de: http://www.redalyc.org/articulo.oa?id=57708106

17. Tardío, J., Morales, R., Aceituno, L., Molina, M. \& Pardo de Santayana, M. (2006). Biodiversidad y etnobotánica en España. Memorias R. Soc. Esp. Hist. Nat., 2ª ép., 9, 2011

18. Trujillo, M. (2010). Plantas útiles en una comunidad indígena Murui-Muinane desplazada a la ciudad de Florencia (Caquetá- Colombia). Mundo Amazónico 1: 267-278.

19. Usma, M.C. (2003). Manejo de flora silvestre aprovechada por la comunidad Wounaan San Bernardo, en el río San Juan (Valle-Chocó), Colombia. Revista Universidad Tecnológica del Choco 2003. 2: 42-57.

20. Yaroslava N. (2002). Guía ilustrada de plantas leñosas útiles de la comunidad San José de Uchupiamonas Provincia Abel Iturralde, Departamento de La Paz, Bolivia. Revista Universidad Católica de San Pablo 2004. 3: 18-26

\section{Conflicto de Intereses}

Los autores declaran no tener ningún conflicto de intereses
|Recibido: 14 de mayo de 2014

Aceptado: 10 de julio de 2014 
